\title{
Synchrotron X-ray Characterization and The Development of a Biomaterials Pipeline at DuPont
}

\author{
J David Londono, Laura Clinger \\ DuPont, Experimental Station, Wilmington DE 19803 \\ j-david.Iondono@dupont.com
}

For over 20 years, the DuPont-Northwestern-Dow Collaborative Access Team (DND-CAT) at the Advanced Photon Source has been supporting the development of a biomaterials pipeline at DuPont. Studies at DND-CAT have enabled elucidation of the structure of these materials in terms of crystallinity, morphology and orientation development during processing, in addition to correlation of microstructure with end-use properties. I will focus on three specific products.

- Sorona ${ }^{\circledR}$ - Eco-efficient Performance Fiber for Home Interiors and Apparel

- Everact $\Omega^{-}$Renewable and High Barrier FDCA-Based Polyesters for Beverage, Food, and Industrial Packaging Markets

- Nuvolve $\AA^{\circledR}$ - An Engineered Polysaccharide for Industrial and Food Applications 